\title{
Beneficial effects of extracts from Lucilia sericata maggots on burn wounds in rats
}

\author{
HAIXU BIAN ${ }^{1,2 *}$, QIAOLI YANG ${ }^{3 *}$, TAO MA ${ }^{4 *}$, WEI LI $^{5 *}$, JIALIN DUAN $^{1 *}$, GUO WEI $^{1}$, \\ XIAOXIAO WU ${ }^{1}$, FEI MU ${ }^{1}$, RUI LIN ${ }^{1}$, AIDONG WEN ${ }^{1}$ and MIAOMIAO XI ${ }^{1}$ \\ ${ }^{1}$ Department of Pharmacy, Xijing Hospital, Fourth Military Medical University, Xi'an, Shaanxi 710032; ${ }^{2}$ Outpatient \\ Department of Xinjiang Forest Corps of Chinese People's Armed Police Force; ${ }^{3}$ Second Pharmacology Laboratory, \\ Institute of Materia Medica; ${ }^{4}$ Department of Pharmacy, Urumqi Stomatology Hospital of Xinjiang; ${ }^{5}$ Department of \\ Pharmacy, Urumqi Maternal and Child Care Hospital, Urumqi, Xinjiang 830000, P.R. China
}

Received March 15, 2016; Accepted March 17, 2017

DOI: $10.3892 / \mathrm{mmr} .2017 .7566$

\begin{abstract}
Lucilia sericata maggots have beneficial properties; however, their protective effects on burn wounds have yet to be fully elucidated. In the present study, a deep second-degree burn rat model was used to investigate the burn wound healing properties of aqueous extract of maggots (MAE). The anti-inflammatory, antioxidative and antibacterial activities were examined. In addition, the protein expression levels of Akt, vascular endothelial growth factor A (VEGFA) and nuclear factor- $\kappa \mathrm{B}(\mathrm{NF}-\kappa \mathrm{B})$ were detected by western blotting. The findings of the present study revealed that MAE treatment increased burn wound healing and hydroxyproline content in the burn-treated rats. A total of seven compounds (MAE-P1-P7) were separated from MAE and a comparative study was performed to identify the major active component. The results demonstrated that MAE-P6 exerted greater antibacterial activity compared with the other compounds. MAE-P6 treatment reduced tissue levels of malondialdehyde, tumor necrosis factor- $\alpha$ and interleukin- 6 , and increased superoxide dismutase activity. Furthermore, MAE-P6 increased the expression levels of VEGFA and reduced NF- $\mathrm{kB}$ expression through Akt, which was verified by treatment with the Akt-specific inhibitor, LY294002. In conclusion, the present study demonstrated that the beneficial effects of MAE on burn wound healing were due to its antibacterial, antioxidative and anti-inflammatory activities. MAE-P6 reduced the release
\end{abstract}

Correspondence to: Professor Aidong Wen or Professor Miaomiao Xi, Department of Pharmacy, Xijing Hospital, Fourth Military Medical University, 127 Changle West Road, Xi'an, Shaanxi 710032, P.R. China E-mail: adwen-2012@hotmail.com

E-mail: miaomiaoxi2014@163.com

*Contributed equally

Key words: aqueous extract of maggots, anti-inflammatory, antioxidative activity, antibacterial activity, burn of inflammatory cytokines via the $\mathrm{Akt} / \mathrm{NF}-\kappa \mathrm{B}$ signaling pathway, and regulated angiogenesis and vasopermeability via the Akt/VEGFA pathway.

\section{Introduction}

The number of patients suffering from burn injuries has increased over time (1); this type of chronic and non-healing wound, and its associated treatment, is considered a major medical and economic issue $(2,3)$. Natural and synthetic drugs have been used to treat burn injuries, in order to identify an efficient method to control the damage. Natural remedies are considered safe and cheap therapeutic alternatives; therefore, the demand for them is increasing in developing countries, as well as in certain developed countries. Various plants or animals with antibacterial and healing properties have been identified as effective treatments for burn injuries (4-6), including Lucilia sericata maggots.

L. sericata maggots have been clinically used to treat decubital necrosis; gastrointestinal problems, such as indigestion and malnutrition; ecthyma and burn injuries since the Ming/Qing dynasty (1368 AD) in China. This therapeutic method became popular worldwide for the treatment of chronic and infected wounds in the 1930s (7). Maggot debridement therapy (MDT) is a therapeutic method that has proven effective for the cleansing of non-healing wounds. Previous comparative clinical trials have investigated the efficacy of MDT $(8,9)$, and demonstrated that MDT is more effective compared with conventional therapies in terms of debridement (8). Paul et al (10) used MDT to clinically treat diabetic foot ulcers; the findings revealed that MDT was effective when used as a debridement technique. In addition, the ward stay time and amputation rate were reduced in MDT-treated patients (10). Maggots have been reported to exert several beneficial effects on wounds, including cleansing, disinfection, debridement and healing $(11,12)$.

Maggot therapy has been adopted as an effective debridement technique for the management of chronic wounds $(13,14)$; however, it remains cosmetically unappealing to the majority of patients and nursing staff, and the maggots themselves have a limited shelf life (15). For more fields to benefit from the use 
of maggots, a switch to contemporary biosurgery, involving the application of maggot-derived substances instead of live insects, should be investigated for future treatments. A previous study isolated and characterized the beneficial molecules in maggot secretions, with the aim of producing them synthetically or as recombinant proteins that may be applied as topical formulations (15). However, a limited number of studies have investigated the beneficial molecules in maggot secretions and the underlying molecular mechanisms $(16,17)$.

In the present study, antimicrobial proteins were extracted and purified from the body of maggots and their biological activity was investigated in vivo. In addition, the underlying molecular mechanisms of action on second-degree burn injury were evaluated.

\section{Materials and methods}

Sterile larvae and crude protein extract. A laboratory colony of L. sericata flies was obtained from Xi'an Furulin Biological Technology Co., Ltd. (Xi'an, China) and sterile maggots were reared in our laboratory, according to a previously described method by Mumcuoglu et al (18). The maggots were 4-5 days old prior to extraction of the proteins.

To extract the active ingredients from the maggots, the maggots were frozen in liquid nitrogen and homogenized using a mortar, subsequently the homogenate was lyophilized and the pulverized material was dissolved in $0.1 \mu \mathrm{g} / \mathrm{ml}$ aprotinin in $0.1 \%$ trifluoroacetic acid $(1: 10 \mathrm{v} / \mathrm{v})$; the suspension was maintained on ice in the dark for $1 \mathrm{~h}$ and centrifuged 3 times at $20,000 \mathrm{x}$ g at $4^{\circ} \mathrm{C}$ for $30 \mathrm{~min}$ to remove residues. The supernatant was collected and stored at $-80^{\circ} \mathrm{C}$ until further use.

Purification of the crude protein extract. To purify the crude extract, the Sephadex G-50 fine column (26x1,000 mm; GE Healthcare Life Sciences, Chalfont, UK) was used and the extract was eluted at $2 \mathrm{ml} / \mathrm{min}$. Eluted components were collected corresponding to the absorbance at $280 \mathrm{~nm}$ and lyophilized. To analyze the proteins, SDS-PAGE was conducted; MAE samples were separated on a 5.0\% stacking gel and $12.5 \%$ separation gel, followed by Coomassie staining (Nanjing Jiancheng Bioengineering Institute).To determine the antibacterial activity of the extract, and for the performance of other bioassays, the fractions were dissolved in water to $50 \mu \mathrm{g} / \mathrm{ml}$.

Antibacterial tests. Antibacterial activity was tested using a method described by Huberman (19). Briefly, Escherichia coli (ATCC 25922) was purchased from the China Center of Type Culture Collection (Wuhan, China). E. coli was cultured in $20 \mathrm{ml}$ tryptic soy broth (TSB; BD Biosciences, Franklin Lakes, NJ, USA) at $30^{\circ} \mathrm{C}$ for $17 \mathrm{~h}$. Subsequently, $100 \mu \mathrm{l}$ culture was mixed with $10 \mathrm{ml}$ TSB and incubated for a further $4 \mathrm{~h}$ (density $\sim 2 \times 10^{8} / \mathrm{ml}$ ). Nutrient agar (Oxoid, Ltd., Basingstoke, UK) was inoculated with $10 \mu \mathrm{l}$ bacterial culture, at a final density of $4 \times 10^{4} / \mathrm{ml}$. After gentle mixing, the agar was immediately distributed into Petri dishes $(85-\mathrm{mm})$. After cooling and solidification, holes (4-mm-diameter) were bored into the agar, and $10 \mu \mathrm{l}$ samples [aqueous extract of maggots (MAE) P1-P7] were loaded into the holes. Sterile ultrapure water was included as a control. Plates were incubated at $30^{\circ} \mathrm{C}$ overnight and the diameters of bacterial growth inhibition were measured.

Burn injury model and drug administration. Male Wistar rats ( $n=100$; age, 20 \pm 1 weeks; weight, 200-250 g) were provided by the Experimental Animal Center of the Fourth Military Medical University (Xi'an, China). Animals were housed in a room at a controlled temperature $\left(25^{\circ} \mathrm{C}\right)$ under a $12 \mathrm{~h} \mathrm{light} / \mathrm{dark}$ cycle with free access to food and water. The present study was approved by the Ethics Committee of the Fourth Military Medical University.

Rats were attached to a homemade wooden support and anesthetized by $100 \mathrm{mg} / \mathrm{kg}$ ketamine and $8 \mathrm{mg} / \mathrm{kg}$ xylazine through intraperitoneal injection. Back hair was removed and the exposed skin was immersed in $98^{\circ} \mathrm{C}$ water for $15 \mathrm{sec}$ to induce a second-degree burn. A group of animals $(n=8)$ was not burned and served as controls. Jingwanhong ointment (JWH; Tianjin Darentang Jingwanhong Pharmaceutical Co., Ltd., Tianjin, China) was used as a positive control. The animals were divided into the following four groups ( $n=8 /$ group): i) Control group; ii) burn group (model); iii) burn + MAE (0.2 g per wound) group; and iv) burn + JWH $(0.2$ g per wound) group. All the tested medicines were applied on the surface of the wound slowly. The wounds were treated every $24 \mathrm{~h}$ for 14 days. To evaluate effects of MAE-P1-P7 on burn wound, MAE-P1-P7 (0.2 g per wound; $\mathrm{n}=8$ rats/treatment) was administered to rats in the burn + MAE group. In some experiments, LY294002 (Sigma-Aldrich; Merck KGaA, Darmstadt, Germany) was intraperitoneally administered together with MAE-P6, at a dose of $0.3 \mathrm{mg} / \mathrm{kg}$. The burn wound area was observed after all treatments on the 14th day and the rats were anesthetized with ketamine $(75 \mathrm{mg} / \mathrm{kg})$ and sacrificed by decapitation. Blood was subsequently collected, and wound tissues were collected and frozen at $-80^{\circ} \mathrm{C}$ until use.

Determination of hydroxyproline levels. A hydroxyproline assay was used to quantify collagen deposition in the wound area. Tissue samples $(7 \mathrm{mg}$ ) were obtained, subcutaneous fat was removed, and they were incubated at $60^{\circ} \mathrm{C}$ in open microtubes for $20 \mathrm{~h}$ to obtain the dry weight. Subsequently, the samples were hydrolyzed with $6 \mathrm{~mol} / \mathrm{H} \mathrm{HCl}$ by stirring for $15 \mathrm{~h}$ at $120^{\circ} \mathrm{C}$. The lysate was diluted with deionized water and neutralized with $\mathrm{NaOH}$. Subsequently, the lysate was centrifuged at $1,000 \mathrm{x} \mathrm{g}$ at $25^{\circ} \mathrm{C}$ for $15 \mathrm{~min}$, and the supernatant was collected for hydroxyproline assay. The hydroxyproline levels were quantified using the hydroxyproline assay kit (catalog no. A030-1; Nanjing Jiancheng Bioengineering Institute), according to the manufacturer's protocol. Hydroxyproline levels were expressed as $\mathrm{mg} / \mathrm{g}$ of tissue.

Determination of malondialdehyde (MDA) levels. MDA is an index of lipid peroxidation. The MDA level was quantified using a commercial kit (catalog no. A003; Nanjing Jiancheng Bioengineering Institute), which results in the formation of thiobarbituric acid-reactive substances during an acid-heating reaction. To precipitate proteins, tissue samples were mixed with $600 \mu 110 \%$ (w/v) trichloroacetic acid and then centrifuged at $1,000 \times \mathrm{g}$ for $10 \mathrm{~min}$ at $25^{\circ} \mathrm{C}$. Supernatants were collected and treated with an equal volume of $0.67 \%$ thiobarbituric acid in a boiling water bath for $15 \mathrm{~min}$. After cooling for 
$5 \mathrm{~min}$ the reaction products were measured at $535 \mathrm{~nm}$. MDA $(\mathrm{nmol} / \mathrm{mg})=\left(\mathrm{OD}_{\text {test }}-\mathrm{OD}_{\text {blank }}\right) /\left(\mathrm{OD}_{\text {standard }}-\mathrm{OD}_{\text {blank }}\right) \times 10 /$ protein concentration, where OD refers to optical density. The level of MDA was expressed as nmol/mg of protein.

Determination of superoxide dismutase (SOD) levels. The enzyme activity of SOD was quantified using a spectrophotometric assay kit (catalog no. A001-3; Nanjing Jiancheng Bioengineering Institute), according to the manufacturer's protocol. Briefly, the proteins in the tissue samples were extracted using a protein extraction kit (catalog no. P0033; Beyotime Institute of Biotechnology, Nanjing, China). The protein samples were incubated with the provided radical detector reagent and xanthine oxidase was subsequently added to initiate the reaction. Following incubation at room temperature for $20 \mathrm{~min}$ with agitation, the reaction system was measured at $450 \mathrm{~nm}$. SOD (U/mg prot $)=\left(\mathrm{OD}_{\text {standard }}-\mathrm{OD}_{\text {test }}\right) / O D_{\text {standard }} / 0.5 /$ protein concentration, where OD refers to optical density. The levels of SOD were expressed as U/mg of protein.

Determination of serum levels of tumor necrosis factor- $\alpha$ (TNF- $\alpha)$ and interleukin-6 (IL-6). Blood samples were collected 14 days after burns. To separate serum, blood samples were maintained at room temperature for $1 \mathrm{~h}$, and were centrifuged at $1,000 \times \mathrm{g}$ for $10 \mathrm{~min}$ at $25^{\circ} \mathrm{C}$. Serum levels of TNF- $\alpha$ and IL- 6 were quantified using rat TNF- $\alpha$ ELISA kit (catalog no. PT516) and rat IL-6 ELISA kit (catalog no. PI328; Beyotime Institute of Biotechnology), according to the manufacturer's protocols. The level was expressed as ng/l.

Western blot analysis. Proteins in excised tissues were homogenized using a protein extraction kit (catalog no. P0033; Beyotime Institute of Biotechnology) and were centrifuged at $12,000 \mathrm{x} \mathrm{g}$ for $15 \mathrm{~min}$ at $4^{\circ} \mathrm{C}$. Protein concentration was determined using a bicinchoninic acid kit (catalog no. A045-3; Nanjing Jiancheng Bioengineering Institute). The supernatant was then collected, mixed with $2 \mathrm{X}$ SDS loading buffer and denatured at $100^{\circ} \mathrm{C}$ for $5 \mathrm{~min}$. Equal amounts of protein $(40 \mu \mathrm{g})$ were separated by $10 \%$ SDS-PAGE. Subsequently the proteins were transferred to nitrocellulose membranes (Pall Corporation, New York, NY, USA), which were blocked with $5 \%$ non-fat dry milk in $1 \mathrm{X}$ Tris-buffered saline. The membranes were then incubated with Akt (catalog no. ab79360), phosphorylated (p)-Akt (catalog no. ab81283), nuclear factor (NF) $-\kappa B$ (catalog no. ab28856) and vascular endothelial growth factor A (VEGFA; catalog no. ab51745) rabbit anti-rat monoclonal antibodies (1:1,000; Abcam, Cambridge, UK) at $4^{\circ} \mathrm{C}$ for $8 \mathrm{~h}$ and were then incubated with horseradish peroxidase-conjugated anti-rabbit secondary antibody (catalog no. sc-2357; 1:5,000; Santa Cruz Biotechnology, Inc., Dallas, TX, USA) or horseradish peroxidase-conjugated anti-mouse secondary antibody (catalog no. ab97255; 1:5,000; Abcam). $\beta$-actin (1:1,000; catalog no. ab8226; Abcam) was used as a control. All bands were detected by enhanced chemiluminescence (ECL) using the ECL Western Blot detection kit (GE Healthcare Life Sciences). Western blots were semi-quantified by densitometric analysis using Quantity One 1-D software (Bio-Rad Laboratories, Inc., Hercules, CA, USA).
Analysis of microvessel density (MVD) and VEGFA expression. After the rats were anesthetized with ketamine, 4\% paraformaldehyde was routinely perfused through left ventricle for $1 \mathrm{~h}$, and wound tissue was collected and fixed in $4 \%$ paraformaldehyde for $24 \mathrm{~h}$. Sections $(5.0 \mu \mathrm{m})$ were obtained from paraffin-embedded tissues, and were then deparaffinized and rehydrated. To block endogenous peroxidase activity, sections were treated with $3 \%{ }_{2} \mathrm{O}_{2}$ methanol solution for $10 \mathrm{~min}$ at room temperature. For antigen retrieval, sections were boiled in $0.01 \mathrm{~mol} / 1$ citric acid for $20 \mathrm{~min}$ and blocked with $3 \%$ skim milk powder solution for $30 \mathrm{~min}$ at $37^{\circ} \mathrm{C}$. The sections were then washed with PBS and incubated with cluster of differentiation (CD)31 monoclonal antibody (catalog no. sc-376764) or rabbit anti-rat VEGFA (catalog no. sc-152) monoclonal antibody (1:10; Santa Cruz Biotechnology, Inc.) at $37^{\circ} \mathrm{C}$ for $1 \mathrm{~h}$. Sections were washed with PBS, incubated with biotinylated goat anti-mouse (1:100; catalog no. BA1001) and biotinylated goat anti-rabbit secondary antibody (1:100; catalog no. BA1003; Boster Biological Technology Co., Ltd., Zhongshan, China) for $30 \mathrm{~min}$ at $37^{\circ} \mathrm{C}$ and were subsequently incubated with horseradish peroxidase-labeled streptavidin (catalog no. BA1088; Boster Biological Technology Co., Ltd.) for $30 \mathrm{~min}$ at $37^{\circ} \mathrm{C}$. Sections were stained with 3,3'-diaminobenzidine $/ \mathrm{H}_{2} \mathrm{O}_{2}$ and hematoxylin, dehydrated, cleared and mounted for viewing. The quantity of CD31-positive small vessels was observed under a microscope (x400 magnification; DM2500; Leica Microsystems GmbH, Wetzlar, Germany) to determine MVD. The number of counted microvessels per $\mathrm{mm}^{2}$ was considered the MVD. MVD of a sample was calculated as the average MVD of five selected areas. An additional five areas were randomly selected for VEGFA analysis. To calculate VEGFA expression, integrated optical density and density mean of positive expression were used; the images were analyzed with Image Pro Plus version 6.0 (Media Cybernetics, Inc., Rockville, MD, USA). Data were recorded as fold-change.

Statistical analysis. Each experiment was repeated at least three times. Data are presented as the mean \pm standard deviation. SPSS version 18.0 (SPSS Inc., Chicago, IL, USA) was used to analyze the data. Statistically significant differences between treatment groups were determined by one-way analysis of variance, followed by Tukey test for multiple comparisons between groups. $\mathrm{P}<0.05$ was considered to indicate a statistically significant difference.

\section{Results}

$M A E$ reduces the size of the wound area. To evaluate the effects of MAE treatment on burn wound closure, wound contraction was measured. As presented in Fig. 1A, an increase in wound-healing activity was observed in the rats treated with MAE and JWH, as compared with the burn model rats that did not receive treatment. In addition, it was demonstrated that the wound-healing activity of MAE was greater compared with JWH.

Hydroxyproline, which is integral to collagen fibers, may promote wound healing in tissue injury (20). In various wound models, an increase in hydroxyproline content may indicate increased collagen synthesis. In the burn wound model, the 


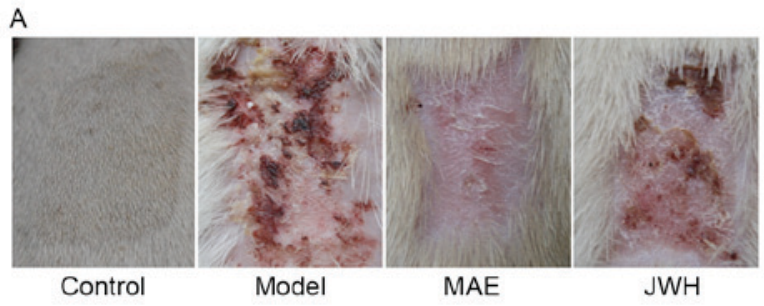

B

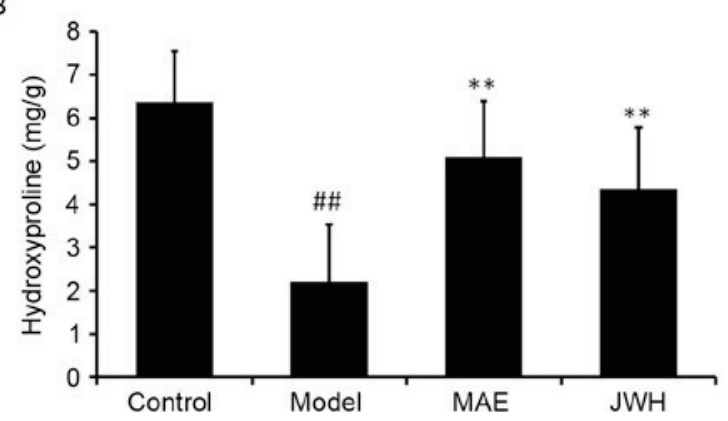

Figure 1. MAE exerted a burn wound healing effect. (A) Wound appearance following 14 days of treatment with MAE. MAE increased wound healing compared with the model group. (B) Effects of MAE on hydroxyproline levels in the burn wound model. Data are expressed as the mean \pm standard deviation. ${ }^{\# \#} \mathrm{P}<0.05$ vs. the control group, ${ }^{* *} \mathrm{P}<0.05$ vs. the model group. MAE, aqueous extract of maggots; JWH, Jingwanhong ointment.

level of hydroxyproline in granulation tissue was significantly reduced compared with in the control group (Fig. $1 \mathrm{~B} ; \mathrm{P}<0.05$ ). However, MAE and JWH treatment significantly increased the hydroxyproline level compared with the model group $(\mathrm{P}<0.05)$. These findings suggested that MAE may be an effective treatment option for second-degree burns.

Partial purification and characterization of the antibacterial components of MAE. In order to identify the major active ingredients in MAE, Sephadex G-50 fine column was previously used (21). Different components were identified according to their UV absorption characteristics (Fig. 2A). Finally, a total of 7 compounds were collected. Antibacterial tests demonstrated that compound 6 (MAE-P6) had a high antibacterial activity, whereas the remaining compounds had little or no antibacterial activity (Fig. 2B). Examination of these fractions by SDS-PAGE revealed that the majority of proteins in the MAE-P6 were $<20 \mathrm{kDa}$ molecular weight (Fig. 2C). In order to determine if the various compounds exert anti-burn activity, MAE-P1-P7 were used to treat rats in the burn wound model group. The results demonstrated that MAE-P6 treatment significantly increased hydroxyproline levels compared with the burn model $(\mathrm{P}<0.05)$; however, no significant difference was identified between the remaining compounds and the burn model (Fig. 2D). Therefore, MAE-P6 may be responsible for the antibacterial activity and healing properties of MAE. MAE-P6 was used for the subsequent experiments.

Effects of MAE on oxidative damage and inflammation. High intracellular levels of reactive oxygen species (ROS), which occur during the initial stages following a burn injury, have been identified as the inducers of oxidative damage. A previous study suggested that topical application of antioxidants may provide a photoprotective effect and may be considered an effective strategy for reducing burn-induced oxidative damage to the skin (22). The level of MDA is an indication of lipid peroxidation in tissues. The findings of the present study demonstrated that treatment with MAE-P6 significantly reduced the burn-mediated increase of epidermal MDA when compared with the model group (Fig. 3A; $\mathrm{P}<0.05)$. Following a burn injury, inflammatory cells are recruited to the wound site, initiating the inflammatory stage of the healing process (23). The present study examined the effects of MAE-P6 on burn-induced expression levels of cytokines TNF- $\alpha$ and IL-6. A significant increase in the levels of TNF- $\alpha$ and IL- 6 was observed following the administration of the burn compared with the control group (Fig. 3B and C; $\mathrm{P}<0.05)$. However, topical application of MAE-P6 for 14 days post-burn significantly reduced the levels of IL- 6 and TNF- $\alpha$ compared with in the burn model group (Fig. 3B and C; $\mathrm{P}<0.05)$. SOD acts as the first line of defense against superoxide anions $\left(\mathrm{O}^{2} \bullet\right.$ ), by dismutating these anions to $\mathrm{H}_{2} \mathrm{O}_{2}$. SOD activity was significantly reduced in the burn model group compared with the control group (Fig. 3D; $\mathrm{P}<0.05$ ). However, this activity was significantly increased following 14 days of treatment with MAE-P6 when compared with the burn model group (Fig. 3D; $\mathrm{P}<0.05$ ). These findings revealed that MAE-P6 was able to reduce levels of oxidative stress in the rats, potentially limiting the damaging effect of ROS following burn administration.

Effects of MAE on MVD and VEGFA expression. MVD and VEGFA levels in the model group were significantly reduced compared with in the control group 14 days after the burn was administered (Fig. 4; $\mathrm{P}<0.05$ ). MVD and VEGFA levels in the MAE-P6 and JWH groups were significantly higher compared with in the model group (Fig. 4; P<0.05). In addition, the MVD and VEGFA levels in the MAE-P6 group were slightly higher when compared with the JWH group, but had no significant difference.

Effects of MAE on the expression levels of Akt, NF- $\mathrm{B} B$ and $V E G F A$. The Akt signaling pathway regulates numerous physiological processes associated with apoptosis, oxidative stress and inflammation (24). Therefore, the present study determined the effects of topical administration of MAE-P6 on the phosphorylation of Akt following burn administration using western blot analysis. The findings indicated that phosphorylation of Akt was significantly reduced post-burn, as compared with in the control group (Fig. 5; P<0.05). Conversely, MAE-P6 treatment significantly increased phosphorylation of Akt compared with the burn model group (Fig. 5; $\mathrm{P}<0.05$ ).

$\mathrm{NF}-\kappa \mathrm{B}$ is a downstream target of the Akt signal transduction pathway. Western blot analysis indicated that the expression of $\mathrm{NF}-\kappa \mathrm{B}$ was significantly increased in the burn model group compared with in the control group (Fig. 5; $\mathrm{P}<0.05)$. Topical application of MAE-P6 significantly reduced the burn-induced increase in $\mathrm{NF}-\kappa \mathrm{B}$ expression. In addition, the effects of MAE-P6 on the possible regulation of VEGFA signaling were investigated. MAE-P6 reversed the downregulation of VEGFA protein expression, which occurred following burn exposure (Fig. 5). Furthermore, inhibition of Akt by LY294002 eliminated these effects. These findings indicated that MAE-P6 possibly inhibited NF- $\mathrm{NB}$ expression 


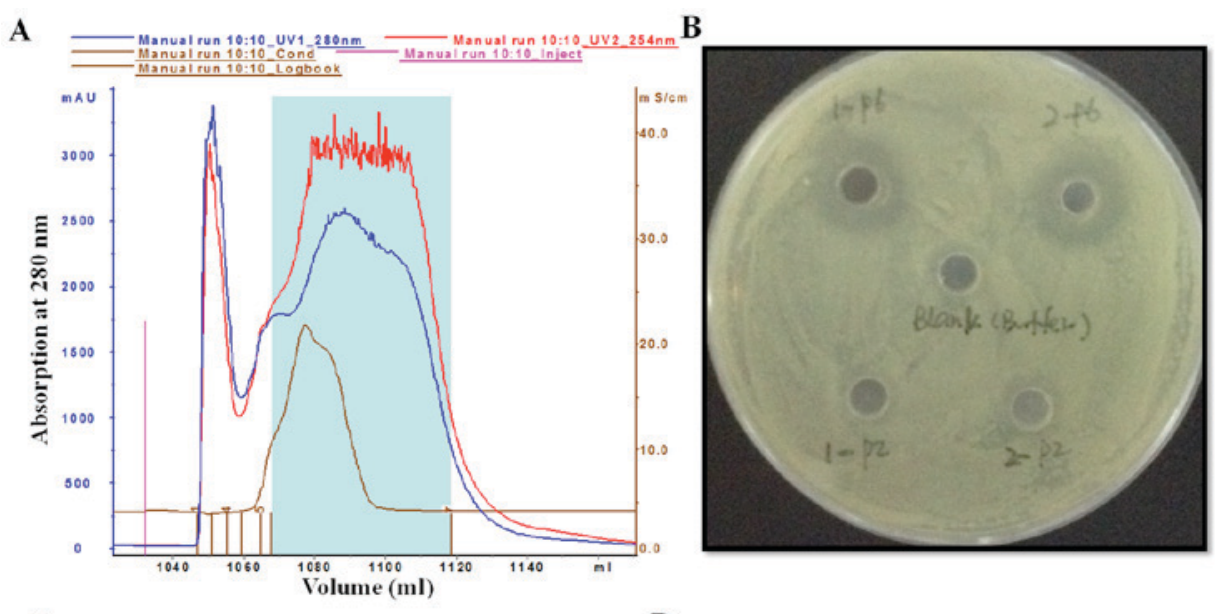

C

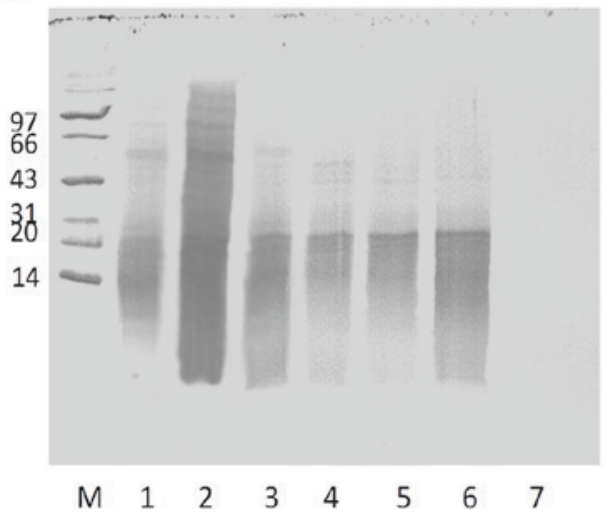

D

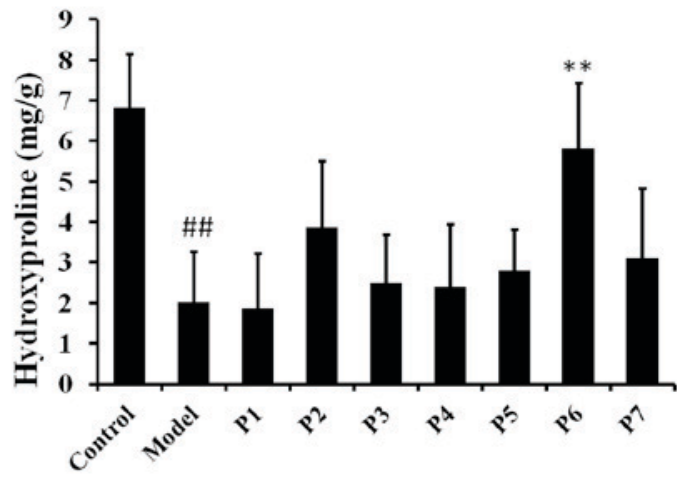

Figure 2. Partial purification and characterization of antibacterial components of MAE. (A) UV absorption characteristics of the various components. (B) Antibacterial tests. Various components (P1-P7) were tested on Escherichia coli (dose, $50 \mu \mathrm{g} / \mathrm{ml}$ ). The antibacterial activity of P2 and P6 is presented (C) SDS-PAGE analysis of the different components. Lane 1, P1; lane 2, P2; lane 3, P3; lane 4, P4; lane 5, P5; lane 6, P6; lane 7, P7. (D) Effects of MAE P1-P7 on hydroxyproline levels in the burn wound model. Data are expressed as the mean \pm standard deviation. ${ }^{\# \#} \mathrm{P}<0.05$ vs. the control group, ${ }^{* *} \mathrm{P}<0.05$ vs. the model group. MAE, aqueous extract of maggots.

A

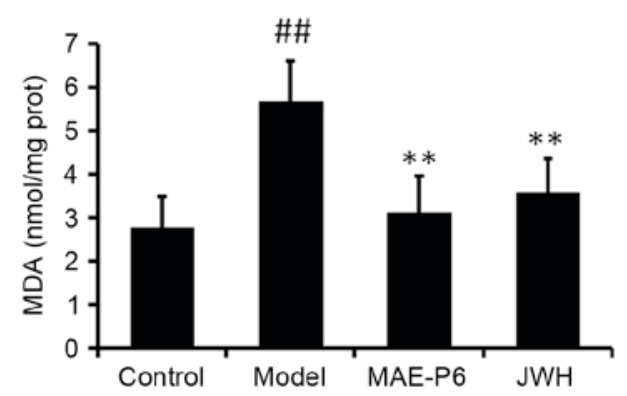

C

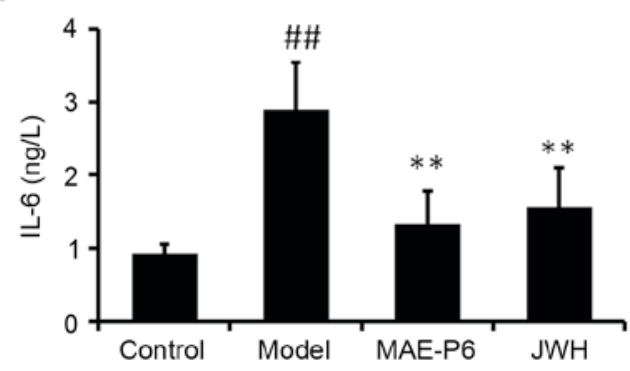

B

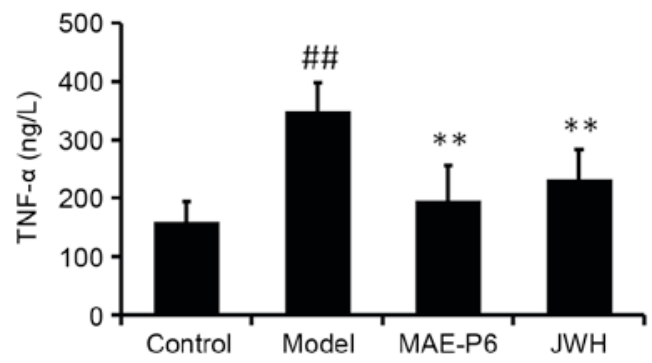

D

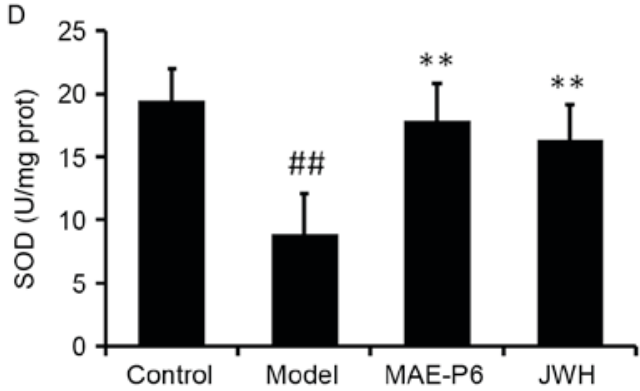

Figure 3. Effects of MAE-P6 on oxidative damage and inflammation. The levels of (A) MDA, (B) TNF- $\alpha$ and (C) IL-6 were increased, whereas the levels of (D) SOD were decreased in the burn model. Application of MAE-P6 following burn treatment led to a significant increase in the activity of SOD and a reduction in the levels of MDA, TNF- $\alpha$ and IL- 6 after 14 days of treatment. Data are expressed as the mean \pm standard deviation. ${ }^{\# \prime} \mathrm{P}<0.05$ vs. the control group, ${ }^{* *} \mathrm{P}<0.05$ vs. the model group. MDA, malondialdehyde; MAE-P6, aqueous extract of maggots-compound 6; JWH, Jingwanhong ointment; TNF- $\alpha$, tumor necrosis factor- $\alpha$; IL-6, interleukin-6; SOD, superoxide dismutase. 

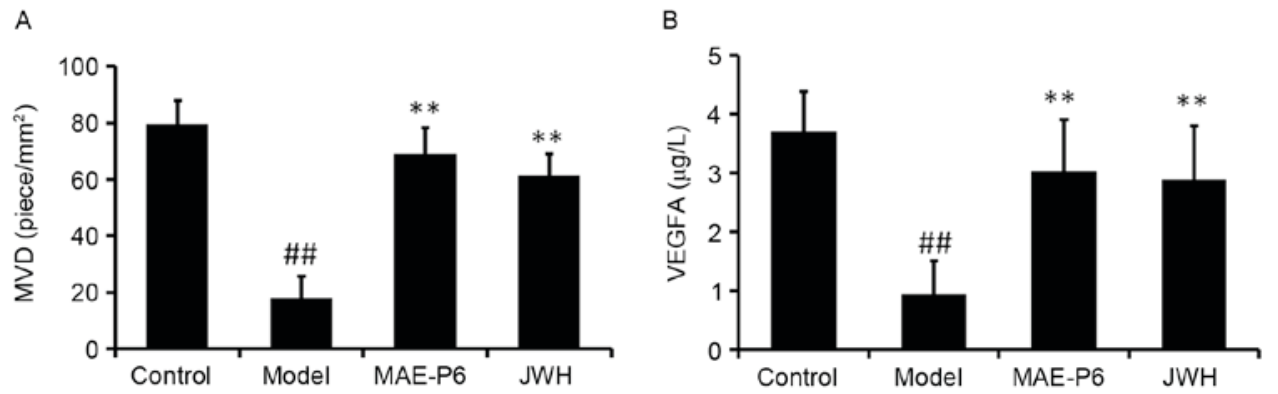

Figure 4. Effects of MAE-P6 on MVD and VEGFA expression in the wound tissues. Wound tissue sections were collected 14 days after burning. (A) MVD and (B) VEGFA were detected using anti-rat cluster of differentiation 31 monoclonal antibody and mouse anti-rat VEGFA monoclonal antibody, respectively. The number of microvessels counted per $\mathrm{mm}^{2}$ was considered MVD. For VEGFA, the images were analyzed using Image Pro Plus version 6.0. Data are expressed as the mean \pm standard deviation. ${ }^{\# \#} \mathrm{P}<0.05$ vs. the control group, ${ }^{* * *} \mathrm{P}<0.05$ vs. the model group. MVD, microvessel density; MAE-P6, aqueous extract of maggots-compound 6; JWH, Jingwanhong ointment; VEGFA, vascular endothelial growth factor A.

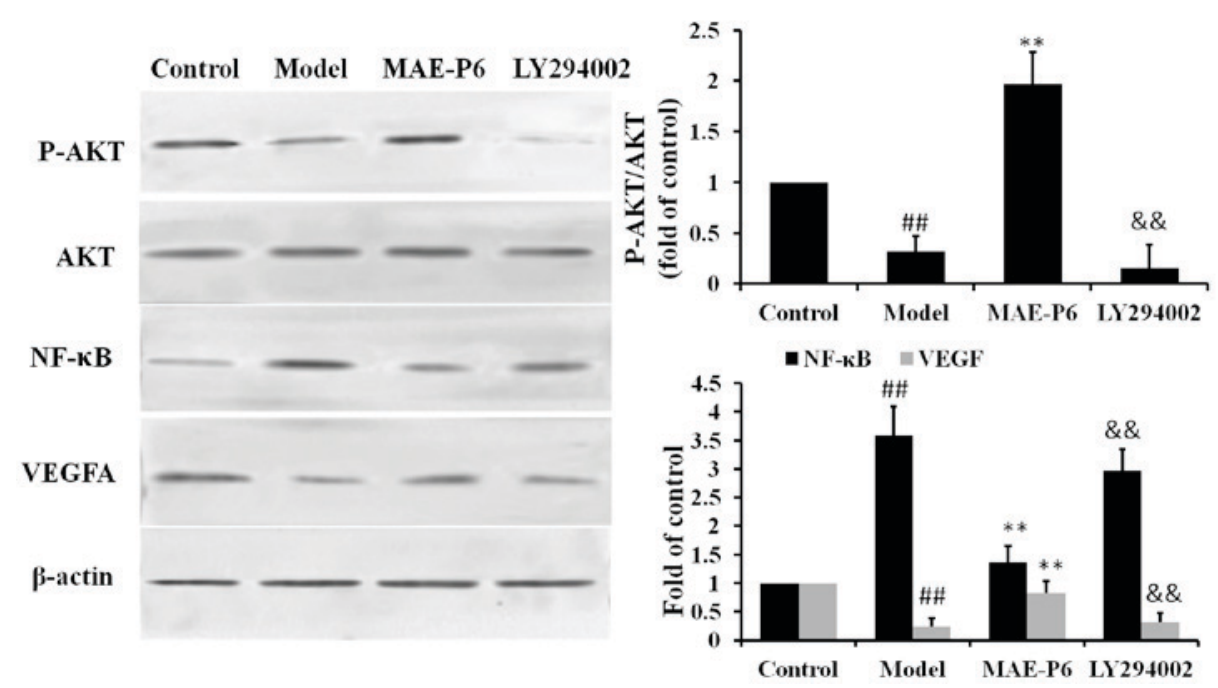

Figure 5. Effects of MAE-P6 on the expression of Akt, NF-кB and VEGFA. Rats were sacrificed 14 days after the burn treatment and tissue lysates were prepared to determine the protein expression levels of Akt, p-Akt, NF- $\mathrm{kB}$ and VEGFA using western blot analysis. Data are expressed as the mean \pm standard deviation. ${ }^{\# \#} \mathrm{P}<0.05$ vs. the control group, ${ }^{* *} \mathrm{P}<0.05$ vs. the model group, ${ }^{\text {\&\&}} \mathrm{P}<0.05$ vs. the MAE-P6 group. p-Akt, phosphorylated-Akt; NF-kB, nuclear factor- $\mathrm{kB}$; VEGFA, vascular endothelial growth factor A; MAE-P6, aqueous extract of maggots-compound 6.

and increased VEGFA expression via phosphorylation of the Akt signaling pathway.

\section{Discussion}

Burns represent a devastating form of trauma, which are associated with severe pain, distress and reduced quality of life for numerous patients worldwide. Thermal injury may severely compromise the skin barrier, affecting important functions, such as protection against infection, thermoregulation and body fluid homeostasis $(25,26)$. Effective treatments associated with wound infection control and wound healing are important for a successful recovery (27). Burn wound healing is categorized into three overlapping stages: i) Inflammation; ii) granulation tissue formation; and iii) remodeling (19). Therefore, acceleration of the rate of tissue regeneration, inhibition of inflammation or oxidative stress, and prevention of wound infection are crucial procedures for burn treatment (28). Natural products, such as insects or plants, may be considered promising for the treatment of burn injuries, and may be used worldwide. A particularly interesting therapeutic strategy in traditional Chinese medicine is the use of maggots, which are the larvae of insects and terrestrial arthropods; however, only a small number of studies regarding this treatment strategy are available $(13,14)$. The present study aimed to determine the burn wound healing and antibacterial properties of MAE in rats, and observed that MAE treatment accelerated wound contraction 14 days after burn administration, indicating the beneficial effects of this therapeutic agent.

Fibroblasts, which are responsible for the deposition, synthesis and remodeling of the extracellular matrix, are the primary cell type involved in the wound healing process (29). At the initiation of wound repairing, fibroblasts undergo in situ proliferation and differentiation, then migrate from the edges of the wound to the wound site, proliferate again and begin to produce collagen (30). In the healing phase, collagen content in the granulation tissue may be quantified by monitoring the concentration of hydroxyproline, which is a marker of collagen biosynthesis (31). Therefore, the levels of hydroxyproline represent the rate of collagen synthesis. In the present study, 14 days after treatment, an increase in hydroxyproline content was observed in the MAE group $(5.11 \pm 1.28 \mathrm{mg} / \mathrm{g}$ dry weight 
of tissue), which was significantly higher compared with in the model group $(2.21 \pm 1.32 \mathrm{mg} / \mathrm{g})$. These findings indicated that collagen synthesis was improved by treatment with MAE. Therefore, the wound healing effects of MAE treatment may be due to increases in collagen content.

Bacterial infections may occur at the early stages of wound healing, which may delay healing, and lead to failure of healing or wound deterioration (31). Pathogenic microorganisms delay wound healing via various mechanisms, including persistent production of inflammatory mediators, induction of tissue hypoxia, production of cytolytic enzymes and free oxygen radicals, and competitive inhibition of nutrient and oxygen levels (32). Therefore, keeping the wound clean in order to avoid infection or maintaining a good bioburden is necessary for successful wound healing. The present study identified seven compounds isolated from MAE and their bacteriostatic action on Gram-negative bacterial growth was evaluated. As the findings of the present study demonstrated, compound P6 (MAE-P6) exerted a significantly greater antibacterial effect compared with the remaining six compounds. Further investigation revealed that MAE-P6 may increase the levels of hydroxyproline in the wound healing model. Therefore, the antibacterial activity of MAE-P6 may reduce the number of pathogens growing on the skin, thus promoting wound healing.

Exposure to a pathogenic microorganism may activate neutrophils, which produce ROS and lead to oxidative stress. In burns, oxidative stress is a pathogenic factor in the inflammatory response; the excessive production of free radicals recruits inflammatory cells and may lead to endothelial dysfunction (33). However, antioxidants, such as SOD, catalase and glutathione, may eliminate free radicals and accelerate the process of burn wound healing. So it is necessary to estimate these antioxidants in granulation tissues. Significant alterations in the antioxidant profile, combined with elevated levels of MDA, which is a marker of fatty acid oxidation, may lead to impaired wound healing in rats (34). In the present study, the levels of SOD and MDA were quantified in the granulation tissue. The results demonstrated that treatment with MAE-P6 significantly increased the activity of SOD, and reduced the levels of MDA compared with in the burn model group. Notably, MAE-P6 treatment also inhibited the expression levels of the inflammatory cytokines IL- 6 and TNF- $\alpha$.

The surface of burn wounds may be anoxic or hypoxic; both conditions limit the growth of ulcerated or injured tissues. Therefore, treatment that may improve the microcirculation of the burn wound may also improve partial ischemia and hypoxia, and promote healing. VEGFA has previously been described as an important stimulator of angiogenesis and vasopermeability, and is produced by macrophages in the hypoxic burn environment, in order to stimulate the migration and proliferation of endothelial cells $(35,36)$. In the present study, the increase in VEGF and MVD levels coincided with a faster decrease of the wound area (Fig. 4), thus suggesting the importance of angiogenic stimulation by MAE-P6 in the overall acceleration of wound healing.

The possible signaling molecules were also investigated in the present study, in order to determine the potential regulatory targets associated with MAE-P6. The phosphorylation of Akt, which is considered a crucial node of the phosphoinositide 3-kinase-Akt pathway, may be regarded as a cell survival factor via the inhibition of apoptosis and downstream $N F-\kappa B$, resulting in the inhibition of inflammation and oxidative stress $(37,38)$. The findings of the present study revealed that the post-burn reduction in p-Akt was accompanied by an increased release of IL- 6 and TNF- $\alpha$, and an increase in the expression levels of $\mathrm{NF}-\kappa \mathrm{B}$. Conversely, MAE-P6 administration led to increased expression levels of p-Akt and reduced $\mathrm{NF}-\kappa \mathrm{B}$ expression levels. Inhibition of Akt with LY294002 attenuated the expression of $\mathrm{p}$-Akt and increased the expression of $\mathrm{NF}-\kappa \mathrm{B}$. Therefore, it may be hypothesized that the Akt/NF- $\kappa \mathrm{B}$ signaling pathway contributes to MAE-P6-induced inflammatory regulation in burn wounds. Furthermore, inhibition of Akt with LY294002 also inhibited the expression of VEGFA, whereas rats treated with MAE-P6 only exhibited increased expression of VEGFA.

In conclusion, to the best of our knowledge, the present study was the first to demonstrate the beneficial effects of MAE on burn wound healing via its antibacterial, antioxidative and anti-inflammatory activities. It is possible that MAE-P6 functions via the $\mathrm{Akt} / \mathrm{NF}-\kappa \mathrm{B}$ signaling pathway to regulate the release of inflammatory cytokines and free radicals. MAE-P6 may also regulate angiogenesis and vasopermeability via the Akt/VEGFA pathway. The findings of the present study suggested that MAE-P6 has multi-target mechanisms for improving burn wound healing, and may provide useful information for the development of burn wound healing treatments.

\section{Acknowledgements}

The present study was supported by the National Science Foundation of China (grant nos. 81470174, 81173514, 81303264, 81403134,81403135 and 81403182) and the Xijing Research Boosting Program (grant no. XJZT14D06).

\section{References}

1. Mogoşanu GD and Grumezescu AM: Natural and synthetic polymers for wounds and burns dressing. Int J Pharm 463: 127-136, 2014.

2. Lay-flurrie K: Honey in wound care: Effects, clinical application and patient benefit. Br J Nurs 17: S30-S36, 2008

3. Riedel K, Ryssel H, Koellensperger E, Germann G and Kremer T: Pathogenesis of chronic wounds. Chirurg 79: 526-534, 2008 (In German).

4. Ghasemi Pirbalouti A: Medicinal plants used in Chaharmahal and Bakhtyari districts. Iran Herba Pol 55: 34-38, 2009.

5. Nayak BS, Isitor G, Davis EM and Pillai GK: The evidence-based wound healing activity of Lawsonia inermis Linn. Phytother Res 21: 827-831, 2007.

6. Bahramsoltani R, Farzaei MH and Rahimi R: Medicinal plants and their natural components as future drugs for the treatment of burn wounds: An integrative review. Arch Dermatol Res 306: 601-617, 2014.

7. Hou L, Shi Y, Zhai P and Le G: Antibacterial activity and in vitro anti-tumor activity of the extract of the larvae of the housefly (Musca domestica). J Ethnopharmacol 111: 227-231, 2007.

8. Sherman RA: Maggot versus conservative debridement therapy for the treatment of pressure ulcers. Wound Repair Regen 4: 208-214, 2002.

9. Dumville JC, Worthy G, Bland JM, Cullum N, Dowson C, Iglesias C, Mitchell JL, Nelson EA, Soares MO and Torgerson DJ; VenUS II team: Larval therapy for leg ulcers (VenUS II): Randomised controlled trial. BMJ 338: b773, 2009.

10. Paul AG, Ahmad NW, Lee HL, Ariff AM, Saranum M, Naicker AS and Osman Z: Maggot debridement therapy with Lucilia cuprina: A comparison with conventional debridement in diabetic foot ulcers. Inter Wound J 6: 39-46, 2009. 
11. Wollina U, Liebold K, Schmidt WD, Hartmann M and Fassler D Biosurgery supports granulation and debridement in chronic wounds-clinical data and remittance spectroscopy measurement. Int J Dermatol 41: 635-639, 2002.

12. Huberman L, Gollop N, Mumcuoglu KY, Block C and Galun R: Antibacterial properties of whole body extracts and haemolymph of Lucilia sericata maggots. J Wound Care 16: 123-127, 2007.

13. Pinheiro MA, Ferraz JB, Junior MA, Moura AD, da Costa ME Costa FJ, Neto VF, Neto RM and Gama RA: Use of maggot therapy for treating a diabetic foot ulcer colonized by multidrug resistant bacteria in Brazil. Indian J Med Res 141: 340-342, 2015.

14. Nigam Y and Morgan C: Does maggot therapy promote wound healing? The clinical and cellular evidence. J Eur Acad Dermatol 30: 776-782, 2016

15. Vilcinskas A: From traditional maggot therapy to modern biosurgery. In: Insect Biotechnology. Vilcinskas A (ed). Dordrecht, Netherlands, Springer, pp67-755, 2011.

16. Bohova J, Majtan J, Majtan V and Takac P: Selective antibiofilm effects of Lucilia sericata larvae secretions/excretions against wound pathogens. Evid Based Complement Alternat Med 2014: 857360, 2014.

17. Hultmark D: Techniques in Insect Immunology. Weisner A Dunphy GB, Marmaras VJ, Morishima I, Sugumaran M and Yamakawa M (eds). SOS Publications, New Jersey, pp103-1075, 1998.

18. Mumcuoglu KY, Ingber A, Gilead L, Stessman J, Friedmann R, Schulman H, Bichucher H, Ioffe-Uspensky I, Miller J, Galun R and Raz I: Maggot therapy for the treatment of intractable wounds. Int J Dermatol 8: 623-627, 1999.

19. Huberman L, Gollop N, Mumcuoglu KY, Block C and Galun R: Antibacterial properties of whole body extracts and haemolymph of Lucilia sericata maggots. J Wound Care 16: 123-127, 2007.

20. Morellini NM, Giles NL, Rea S, Adcroft KF, Falder S, King CE, Dunlop SA, Beazley LD, West AK, Wood FM and Fear MW: Exogenous metallothionein-IIA promotes accelerated healing after a burn wound. Wound Repair Regen 16: 682-690, 2008

21. Lu J and Chen ZW: Isolation, characterization and anti-cancer activity of SK84, a novel glycine-rich antimicrobial peptide from Drosophila virilis. Peptides 31: 44-50, 2010.

22. Sen CK: The general case for redox control of wound repair. Wound Repair Regen 11: 431-438, 2003.

23. Guo S and Dipietro LA: Factors affecting wound healing. J Dent Res 89: 219-229, 2010.

24. Hassan B, Akcakanat A, Holder AM and Meric-Bernstam F Targeting the PI3-kinase/Akt/mTOR signaling pathway. Surg Oncol Clin N Am 22: 641-664, 2013.
25. Hettiaratchy S and Dziewulski P: ABC of burns. BMJ 328: 1366-1368, 2004

26. Church D, Elsayed S, Reid O, Winston B and Lindsay R: Burn wound infections. Clin Microbiol Rev 19: 403-434, 2006.

27. DeSanti L: Pathophysiology and current management of burn injury. Adv Skin Wound Care 18: 323-332, 2005.

28. Rowan MP, Cancio LC, Elster EA, Burmeister DM, Rose LF, Natesan S, Chan RK, Christy RJ and Chung KK: Burn wound healing and treatment: Review and advancements. Critical Care 19: 243, 2015.

29. Hinz B: The myofibroblast: Paradigm for a mechanically active cell. J Biomech 43: 146-155, 2010.

30. Werner S, Krieg T and Smola H: Keratinocyte-fibroblast interactions in wound healing. J Invest Dermatol 127: 998-1008, 2007.

31. Guo S and Dipietro LA: Factors affecting wound healing. J Dent Res 89: 219-229, 2010.

32. Macri L and Clark RA: Tissue engineering for cutaneous wounds: Selecting the proper time and space for growth factors, cells and the extracellular matrix. Skin Pharmacol Physiol 22: 83-93, 2009.

33. Richelle M, Sabatier M, Steiling H and Williamson G: Skin bioavailability of dietary vitamin E, carotenoids, polyphenols, vitamin C, zinc and selenium. Br J Nutr 96: 227-238, 2006.

34. Gupta A, Singh RL and Raghubir R: Antioxidant status during cutaneous wound healing in immunocompromised rats. Mol Cell Biochem 241: 1-7, 2002.

35. Mast BA and Schultz GS: Interactions of cytokines, growth factors and proteases in acute, and chronic wounds. Wound Repair Regen 4: 411-420, 1996.

36. Distler JH, Hirth A, Kurowska-Stolarska M, Gay RE, Gay S and Distler O: Angiogenic and angiostatic factors in the molecular control of angiogenesis. Q J Nucl Med 41: 149-161, 2003.

37. Baehrecke EH: Autophagy: Dual roles in life and death? Nat Rev Mol Cell Biol 6: 505-510, 2005.

38. Sarkar S: Regulation of autophagy by mTOR-dependent and mTOR-independent pathways: Autophagy dysfunction in neurodegenerative diseases and therapeutic application of autophagy enhancers. Biochem Soc Trans 41: 1103-1130, 2013. 